\title{
IDENTIFICATION OF TEMPERATURE PROFILE AND HEAT TRANSFER ON A DIELECTRIC MEMBRANE FOR GAS SENSORS BY "COSMOS" PROGRAM SIMULATION
}

M. Dumitrescu ${ }^{1}$, C. Cobianu ${ }^{1}$, D. Lungu ${ }^{1}$, D. Dascalu ${ }^{1}$, A. Pascu, A. van den Berg ${ }^{3}$, J.G.E. Gardeniers ${ }^{3}$, S. Kolev ${ }^{4}$, C. Dücsö ${ }^{5}$ and I. Barsony ${ }^{5}$

1. Institute of Microtechnology, P.O. Box 27-17, 77550 Bucharest,Romania

2 . "Politehnica" University Bucharest, Mechanics Faculty, Romania

3. MESA Research Institute, University of Twente, Faculty of Electrical

Engineering, P.O. Box 217, 7500 AE Enschede, The Netherlands.

4 . University of Sofia, Faculty of Chemistry, Sofia, Bulgaria

5. MTA-ATKI, H-1525 Budapest, P.O. Box 49, Hungary.

\section{SUMMARY}

The application of commercial 3-D software "COSMOS" for the design and thermal analysis of the low power consumption test structures with dielectric membrane for gas microsensors is presented. Within this work, the simulation provides the estimation of the temperature profile on the active area and the whole membrane including the four bridges and the heating efficienty in the temperature range $20-500^{\circ} \mathrm{C}$. Unravelling of the heat loss mechanisms in terms of radiation, convection, conduction by air and solid materials during heat transfer on the dielectric membrane is reported for the first time as a mean to evaluate by 3-D simulation the contribution of technological processes and lay-out design to the total heat losses.

\section{INTRODUCTION}

In the last years, many efforts have been made to develop gas sensors compatible with silicon integrated circuit (IC) technology /1-5/. Back-side bulk micromachining was initially the standard processing for the silicon or dielectric membrane fabrication, aimed at reducing the electric power consumption necessary for the sensing film heating /1-3/. Front-side bulk micromachining was further used to release the dielectric membrane $/ 4,5 /$ due to its advantage of eliminating the double side alignement for the anizotropic etching of the silicon substrate. In order to increase the IC compatibility of gas sensors, the heating resistor has been made of $\mathrm{p}^{++}$silicon, which survived below the dielectric membrane due to its etch stop properties in anizotropic etch solutions like $\mathrm{KOH}$ or EDP. Within this paper, we try to simulate the thermal regime of a dielectric membrane (heated by a $\mathrm{p}^{++}$resistor) supported by four bridges and to identify the contribution of different heat loss mechanisms to the equilibrium temperature on the heated membrane of the gas microsensor. It will be shown that the robust metallization system which is necessary to assure chemical and electrical stability at relatively high temperatures during sensor operation (for example $450^{\circ} \mathrm{C}$ for $\mathrm{CH}_{4}$ detection) will determine the main heat loss by its thermal conduction. For this purpose, the commercial 3-dimensional "COSMOS" software based on finite element method will be used.

\section{DESCRIPTION OF TEST STRUCTURE AND THERMAL ANALYSIS METHODOLOGY}

The gas sensor which is simulated here is obtained by sol-gel deposition of $\mathrm{SnO}_{2}$ films on a dielectric substrate provided with interdigitated metallic electrodes made of 
$\mathrm{Au} / \mathrm{Ag} / \mathrm{Mo}$. The same type of metallization system is used for the ohmic contact to the meandered $\mathrm{p}^{++}$silicon resistor which survives below the dielectric membrane $(1 \mu \mathrm{m})$ made of $\mathrm{Si}_{3} \mathrm{~N}_{4} / \mathrm{SiO}_{2}$. Front side bulk micromachining based on EDP etching is applied to release a $400 \mu \mathrm{m} \times 400 \mu \mathrm{m}$ membrane supported at its comers by four bridges, each of them with a length of $560 \mu \mathrm{m}$. The active zone of the sensor is located exactly on $\mathrm{p}^{++}$silicon meander.

The sensor geometry including the bulk silicon is divided in 2737 elements having 3104 nodes. The squared dielectric membrane is divided in $21 \times 21$ elements for each type of layer (For example, for the $\mathrm{Si}_{3} \mathrm{~N}_{4}$ layer each element has a parallelepiped shape with the volume of $19.04 \mu \mathrm{m} \times 19.04 \mu \mathrm{m} \times 0.7$ $\mu \mathrm{m}$, where $0.7 \mu \mathrm{m}$ is the thickness of the layer). The mechanical and thermal constants of volume element are loaded in the program. The biased $\mathrm{Si}$ resistor is the source of heat which is injected as heat flux (obtained from the conversion of the electric power applied on each element of resistor) through the 606 nodes of the meander. The bulk silicon is considered to be at $20^{\circ} \mathrm{C}$.

In order to identify the heat lossses due to conduction, convection and radiation during heat transfer on the dielectric membrane for a certain input electric power, the following sequence of thermal simulations was done:

1. Initially, the whole input power was considered to be dissipated on the solid membrane, with no contribution from convection, conduction by air and radiation which were not defined, and therefore the simulation program could not introduce them. This simulation provided the apparent maximum temperature on the membrane, at the node 232 (centre of the membrane).

2. The parameters for convection simulation were introduced in the program by the CEL command defining the beginning element, the face, the film coefficient, the ambient temperature, the ending element and the increment. Thus, for example, the command CEL 442/6/9/20/882/1 was used for evaluation of the convection in air on the upper side of the membrane.

3. The parameters for radiation simulation were introduced in the program (command REL) defining the beginning element, the faces, the view factor, the source temperature, the ending element and the increment. For example, the command REL 444/6/0.8/1/20/880/1 represents the input data for the evaluation of radiation from the $\mathrm{Si}_{3} \mathrm{~N}_{4}$ surface.

4. The parameters for the conduction through air were finally introduced by giving the air conduction properties and defining the surfaces of the silicon pit, below the dielectric membrane.

The final temperature of the membrane is the result of the equilibrium between the input heat flux and the heat losses by the above mechanisms, while the heat loss by the conduction through bridges can be extracted as a result of the difference between the input heat flux and the sum of the above heat loss mechanisms. Thus, the identification of the heat losses at each input power (and temperature on the membrane can be made) and their relative importance can be estimated. Further exploitation of the software will consist in displaying the effect of different geometry parameters on the heat transfer evaluation on the above membrane.

\section{RESULTS AND DISCUSSION}

In Fig. 1 we present the correlation between the maximum temperature on the dielectric membrane and the input electric power applied on the heating resistor.

For a comparison, the results of an analogic calculation of the heat losses on the same dielectric membrane, according to the methodology of Gardeniers /6/ are presented.

From the slope of the linear numeric dependence of the power versus membrane temperature, a heating efficiency of 13.52 ${ }^{\circ} \mathrm{C} / \mathrm{mW}$ of the resistor has been calculated for the specific lay-out and technology of the test structure. 


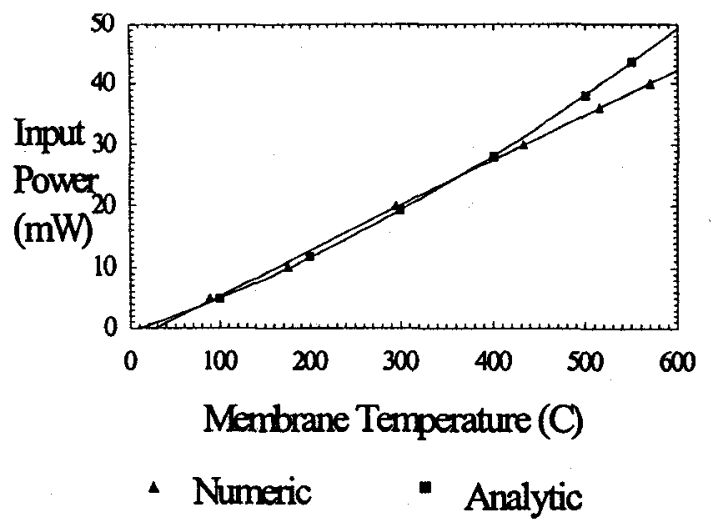

Fig. 1. Input power versus maximum temperature of the membrane for the numeric and analytic evaluation.

In Fig. 2, the temperature profiles on two perpendicular directions including the bridges of the dielectric membrane are shown. The temperature on the bridge is very fast decreasing, reaching the ambient temperature near the bulk silicon. The rapid decrease of the temperature on the membrane might be related to the influence of metallization lay-out in the active area of the sensor.

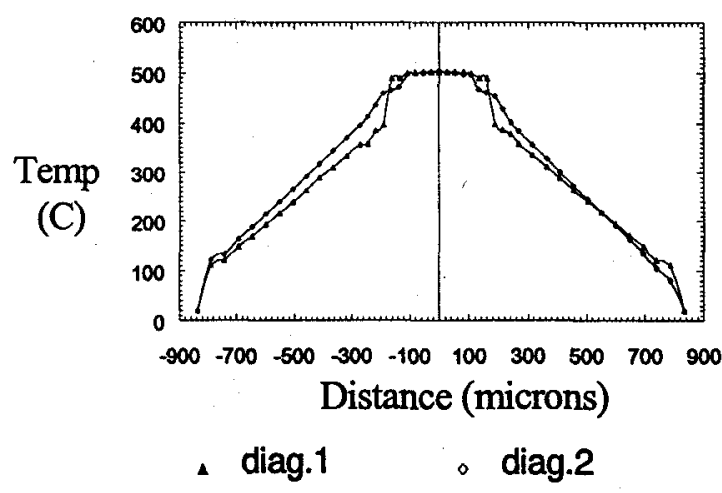

Fig.2 Simulation of temperature profile along the two diagonals of the membrane, including the lengths of the bridges. The membrane is between -282 and $+282, \mu \mathrm{m}$. Input power $=38 \mathrm{~mW}$
In Fig. 3 it is shown the temperature profile in the active area of the membrane, i.e., in the zone of interdigitated metallization made of $\mathrm{Au} / \mathrm{Ag} / \mathrm{Mo}$. One can see that the temperature variation within the sensor area is less than $5^{\circ} \mathrm{C}$, which means of about $1 \%$ of the maximum temperature. We associate this good temperature uniformity on the sensor to the fact that the sensing area is located exactly on the heating resistor.

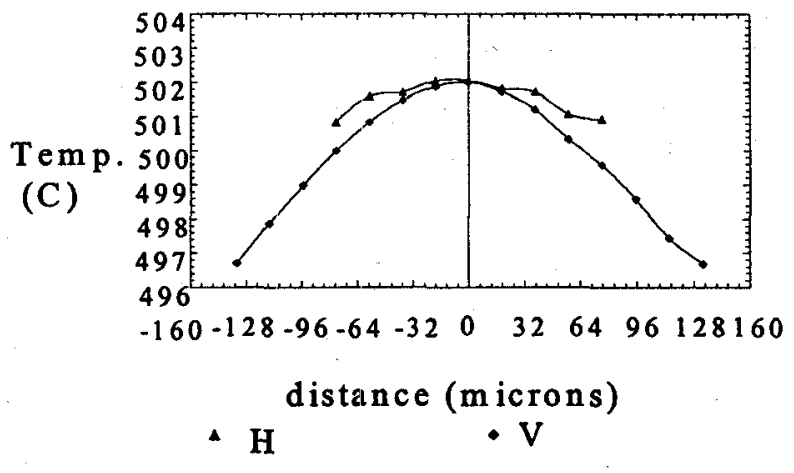

Fig. 3 Temperature profile in the $\mathrm{SnO}_{2}$ sensor area, around its centre. Input power $=38 \mathrm{~mW}$. "H" =horizontal direction; "V" =vertical direction

In order to understand the contribution of the different heat loss mechanism to the total losses and also to have a clear image of the effects of the technological parameters on the heat transfer on the dielectric membrane, we have isolated and identified the contribution of the radiation, convection, conduction through air and bridges in the whole range of applied input power or temperatures on the membrane according to the Fig.1. This procedure was described in the previous section.

Thus, In Fig. 4, we present the unravelling of the heat loss component, so that optimization of the lay-out design or trade-off between the reliability of the sensor technology and the input power necessary to get a certain temperature on the sensor membrane can be analysed. In fact, it is very 
important to mention that the heat lost by conduction through bridges, and especially on

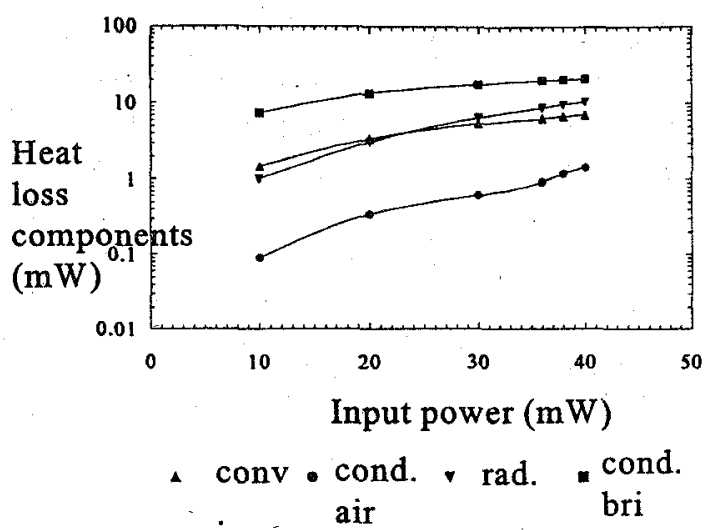

Fig. 4. Separation of heat loss components as a function of the input power on the heating resistor.

the robust metallization has the most important contribution in the whole input power range. This is maximum at $10 \mathrm{~mW}$ (74.2\%) and decreases to $52 \%$ at $40 \mathrm{~mW}$.

Also, one can see the increasing influence of the heat losses by radiation as the temperature on the membrane is increasing. If at $10 \mathrm{~mW}$ of input power, the heat lost by radiation means only $10 \%$ of total heat losses, for an input power of $40 \mathrm{~mW}$, the contribution of radiation is of about $25 \%$, in agreement with the role of this heat loss mechanism at higher temperatures.

\section{CONCLUSIONS}

The commercial 3-D "COSMOS" software represents a powerful and flexible tool for the simulation of the thermal regime of the dielectric membrane used in the test structures for gas sensing applications requiring a low electric power consumption for the heating of the $\mathrm{SnO}_{2}$ sensing film. For our robust test structure, where a reliable metallization system is used to assure a stable sensor operation at temperatures as high as $500^{\circ} \mathrm{C}$, the heating efficiency is estimated to be $13.52{ }^{\circ} \mathrm{C} / \mathrm{mW}$. A temperature uniformity of $1 \%$ in the active sensor area was obtained due to the location of the sensor exactly above the heating resistor. The unravelling of the heat loss components was possible by introducing separately different heat transfer mechanisms in the "COSMOS" program simulation. More than $50 \%$ of heat loss comes from conduction through bridges.

\section{ACKNOWLEDGEMENTS}

This paper was financially supported by the European COPERNICUS project, CP940963, "PORSIS" and the Romanian Ministry of Research.

\section{REFERENCES}

/1/. N. Najafi, K. Wise and J.W. Schwank, IEEE-ED, vol. 41 (1994), 1770-1777.

12/. D-D. Lee, W-Y Chung, T-H Kim and J-M Baek, TRANSDUCERS '95 EUROSENSORS $L X$, Stockholm 1995, paper 210-D5, 827-830.

13/. D. Mutschall, C. Scheibe and E. Obermeier, TRANSDUCERS '95 EUROSENSORS LX, Stockholm 1995, paper 57-PA6, 256-259.

14/. R.E. Cavicchi, J.S. Suehle, K.G. Kreider, M. Gaitan and P. Chaparala, TRANSDUCERS 95 EUROSENSORS IX, Stockholm 1995, paper 209-D5, 823-826.

/5/. S.K.H. Fung, Z. Tang, P.C.H.Chan, J.K.O. Sin and P.W. Cheung, TRANSDUCERS 95EUROSENSORS LX, Stockholm 1995, paper 207-D5, 818-821.

16/. J.G.E. Gardeniers, report at the "PORSIS" meeting, Sofia, January 1996. 